\title{
Schutz von Netzwerken gegen Eingriffe Dritter
}

\section{Verbindung mehrerer Verträge zur Erreichung gemeinsamer Ziele}

Arbeitsteilung einerseits und ehrgeizige technische und wirtschaftliche Ziele andererseits erfordern die Zusammenarbeit mehrerer Beteiligter, um die erstrebten Ziele zu erreichen. Ein wichtiges Mittel hierfür, sind vertragliche Beziehungen zwischen den Beteiligten. Sind mehrere Parteien beteiligt kann ein ganzes Bündel von Verträgen nötig sein, um das Ziel zu erreichen.

Die Zusammenarbeit mehrerer Personen kann in einer Personengesellschaft oder in einer Kapitalgesellschaft erfolgen, wobei alle an ein und demselben Vertrag beteiligt sind. Eine vertragliche Kooperation kann sich aber auch darauf gründen, dass mehrere Verträge zwischen verschiedenen Beteiligten zur Erreichung eines gemeinsamen Ziels miteinander verbunden werden. Man kann dann von einer Zweckgemeinschaft der Verträge sprechen. Dies kann in der Form von Ketten- oder Netzverträgen geschehen.

\section{Kettenverträge}

Verträge, die zum Transport von Gütern mit verschiedenen Verkehrsmitteln nacheinander geschaltet sind, sind ein Beispiel für Kettenverträge ebenso wie Verträge zwischen mehreren Banken, die den Überweisungsbetrag im inländischen wie im grenzüberschreitenden Überweisungsverkehr von der Schuldnerbank über verschiedene Zwischenbanken bis zur Bank des Gläubigers weiterleiten. Als Kettenvertrag kann man auch den Vertrag zwischen Vermieter und Mieter und den anschließenden Vertrag zwischen Mieter und Untermieter einordnen ${ }^{1}$. Die Eigenart solcher Kettenverträge besteht darin, dass die Verträge hintereinander geschaltet sind und der vorgeschaltete Vertrag erfüllt werden muss, damit der nachgeschaltete Vertrag erfüllt werden kann ${ }^{2}$.

\section{Netzverträge}

Bei Netzverträgen existiert ein Systemführer, der Verträge mit mehreren anderen Beteiligten schließt und diese zu einem Angebot an die Kundschaft zusammenstellt ${ }^{3}$. Beispiele für solche Netzverträge sind Zulieferverträge in der Automobilindustrie, die der Hersteller mit mehreren Zulieferern schließt, um aus den gelieferten Teilen das Auto als Endprodukt zu erstellen ${ }^{4}$. Dasselbe trifft auf Bauprojekte zu, bei denen ein

1 Siehe. Marc Amstutz (2006) „Die Verfassung von Vertragsverbindungen“, in diesem Heft I.1.

2 Siehe auch Karl Larenz und Manfred Wolf (2004) Allgemeiner Teil des bürgerlichen Rechts, 9. Aufl., München: Beck, § 23 Rn $127 \mathrm{ff}$.

3 Siehe etwa Larenz und Wolf (Fn. 2) § 23 Rn $130 \mathrm{f}$.

4 Siehe etwa Axel Merz (1992) Qualitätssicherungsvereinbarungen: Zulieferverträge, Vertragstypologie, Risikoverteilung, AGB-Kontrolle, Köln: O. Schmidt; Marina WellenhoferKlein (1999) Zulieferverträge im Privat- und Wirtschaftsrecht, München: Beck. 
Generalunternehmer mit mehreren Subunternehmern kontrahiert, um im Zusammenwirken das Projekt zu erstellen. Ein ähnliches Phänomen zeigt sich, wenn ein Reiseveranstalter Verträge mit mehreren Anbietern, z. B. einem Busunternehmen, einer Luftverkehrsgesellschaft und einem Hotel schließt, um mit den kombinierten Verträgen dem Reisenden eine Gesamtheit von Reiseleistungen gemäß $§ 651$ a BGB anbieten zu können.

Eine weitere Art des Netzvertrags ist das Franchisesystem, in dem der Franchisegeber als Systemführer Verträge mit den Franchisenehmern schließt, die diese in die Lage versetzen, Waren oder Dienstleistungen, die vom Franchisenehmer besorgt und zusammengestellt werden, nach einem einheitlichen Geschäftsmodell unter einer einheitlichen Marke anzubieten ${ }^{5}$.

\section{Kombinationsverträge als neues Schutzobjekt}

Im Folgenden soll nicht die bisher im Mittelpunkt stehende Haftung der Mitglieder des Ketten- oder Netzvertrags für Vertragsverletzungen anderer Mitglieder ins Auge gefasst werden. Vielmehr soll untersucht werden, ob Mitglieder von Ketten- oder Netzverträgen geschützt werden, wenn Dritte das Ketten- oder Netzvertragssystem schädigen. Abgewandelte Beispiele aus der Rechtsprechung sollen dies illustrieren.

\section{Warentestfälle}

Der BGH sieht das Recht am eingerichteten und ausgeübten Gewerbebetrieb als verletzt an, wenn ein Warentest fahrlässig falsche Angaben enthält und dadurch dem Hersteller Schaden zufügt ${ }^{6}$. Geht man davon aus, dass der Test Waren betrifft, die in einem Franchisesystem vertrieben werden und wegen falscher Testergebnisse die Umsätze des Franchisegebers und der Franchisenehmer zurückgehen, stellt sich die Frage, ob nur der Franchisegeber oder auch die Franchisenehmer wegen Verletzung des Rechts am eingerichteten und ausgeübten Gewerbebetrieb Schadensersatz verlangen können oder ob sogar das Franchisesystem als solches als verletztes Rechtsgut anzusehen ist.

\section{Verletzung gewerblicher Schutzrechte}

Dieselben Fragen stellen sich, wenn die Marke, die der Franchisegeber dem System zur Verfügung stellt, verletzt wird oder wenn ein Dritter in Bezug auf die im System genutzten Gewerblichen Schutzrechte zu Unrecht Schutzrechtsverwarnungen aus-

5 Siehe etwa Sven Martinek (1986) »Abzahlungsgesetz und Absatzmittlungsverträge«,7 ZIP, 1440-1449; Gunther Teubner (1990) » Verbund <, >Verband oder >Verkehr $<$ ? Zur Außenhaftung von Franchising-Systemen«, 154 ZHR, 295-324, 305 ff.; Bernd Haager (1999) »Die Entwicklung des Franchiserechts in den Jahren 1997 und 1998«, 52 NJW, 2081-2086, und ders. (2002) »Die Entwicklung des Franchiserechts in den Jahren 1999, 2000 und 2001«, 55 NJW, 1463-1475.

6 Siehe z.B. BGH NJW 1997, 2593, 2595. 
spricht ${ }^{7}$. Kann dann nur der Franchisegeber als Inhaber der Rechte oder können auch die Franchisenehmer Schadensersatz verlangen?

\section{Streikfälle}

Weitere Fälle bilden die Streikfälle, in denen ein Unternehmen infolge eines rechtswidrigen Streiks Produktionsausfälle erleidet. Das BAG hat entschieden, dass durch illegale Streiks das Recht am eingerichteten und ausgeübten Gewerbebetrieb verletzt wird und das betroffene Unternehmen Schadensersatz verlangen kann ${ }^{8}$. Dabei stellt sich die Frage, ob bei einem Netzwerk von Verträgen nur das bestreikte Unternehmen oder auch die im Netzwerk verbundenen Unternehmen Schadensersatz verlangen können, weil z. B. durch den Streik beim Hersteller oder bei einem Zulieferer auch die anderen Mitglieder des Netzwerks betroffen werden können, wenn durch den Ausfall eines Produkts die ganze Produktion zum Stillstand kommt. Macht es einen Unterschied, wenn bei einem Reisevertrag durch den Streik bei einem Leistungserbringer die Reise ausfällt und dadurch auch die anderen beteiligten Unternehmen geschädigt werden?

\section{Situation bei Kettenverträgen}

Was hat zu gelten, wenn an Stelle von Netzverträgen ein System von Kettenverträgen betroffen ist, wie z. B. wenn eine Transportkette durch den Unfall eines Transportmittels oder eine Überweisungskette im Banksystem durch einen Computerwurm unterbrochen wird? Kann nur das direkt betroffene Unternehmen oder auch die anderen Mitglieder der Vertragskette Schadensersatz verlangen?

\section{Unternehmensschutz im System des deutschem Rechts}

Ausgehend vom deutschen System des Rechts der unerlaubten Handlung, das Schadensersatz nur bei Verletzung eines absoluten Rechts (§ 823 I BGB), bei Verletzung eines Schutzgesetzes ( $\$ 823$ II BGB) oder bei vorsätzlicher sittenwidriger Schädigung ( 826 BGB) gewährt, muss grundsätzlich ein absolutes Recht verletzt sein, wenn in den genannten Fällen ein Schadensersatzanspruch bestehen soll.

Im französischen und englischen Recht bewerten die Richter bei Anwendung der dort geltenden Generalklausel die Rechtswidrigkeit des Verhaltens des Schädigers, ohne dass ihnen vom Gesetz weitere Kriterien zur Verfügung gestellt werden. Im deutschen Recht gibt dagegen, wenn kein Schutzgesetz vorhanden ist, die Verletzung eines absoluten Rechts einen Anhaltspunkt für ein rechtswidriges Verhalten, das bei Fehlen eines Rechtfertigungsgrundes die Grundlage für einen Schadensersatzanspruch bildet. Davon ausgehend hat schon das Reichsgericht ${ }^{9}$ und ihm folgend der $\mathrm{BGH}^{10}$ das Recht

7 Dazu etwa BGHZ 38, 205 und zuletzt BGH GSZ vom 15.7.2005 GSZ 1/04 = NJW 2005, 3141.

8 BAG NJW 1978, 2114; 1989, 1881.

9 Siehe RGZ 58, 24; 73, 107.

10 BGHZ 8, 142; 36, 18; 45, 296, 307. 
am eingerichteten und ausgeübten Gewerbebetrieb oder, wie es auch genannt wird, das Recht am Unternehmen geschaffen, um ein Recht zu haben, das im Falle seiner Verletzung die Grundlage für die Beurteilung des rechtswidrigen Verhaltens sein kann.

Zwar ist die Anerkennung dieses Rechts nicht unbestritten. Manche lehnen ein solches Recht am Unternehmen ab und gewähren Schutz nur nach dem UWG oder bei vorsätzlicher sittenwidriger Schädigung $(\S 826 \mathrm{BGB})^{11}$. Dieser Schutz ist aber unzureichend. Das UWG findet keine Anwendung, wenn zwischen den Beteiligten kein Wettbewerbsverhältnis besteht und $\S 826$ BGB gibt keinen Schutz bei fahrlässiger Schädigung. Das Recht am eingerichteten und ausgeübten Gewerbebetrieb ist deshalb mit den Gerichten anzuerkennen, um einen adäquaten Schutz für die Unternehmenstätigkeit zu gewähren ${ }^{12}$.

Das Recht am eingerichteten und ausgeübten Gewerbebetrieb bringt Probleme insoweit mit sich, als seine Funktion, im Falle seiner Verletzung die Rechtswidrigkeit zu indizieren, nicht sehr stark ausgebildet ist. Die normalen Rechtfertigungsgründe reichen nicht aus, um das rechtswidrige Verhalten vom rechtmäßigen Verhalten, insbesondere im Hinblick auf die Wettbewerbs- und Meinungsfreiheit abzugrenzen. Deshalb muss bei Anerkennung eines Rechts am eingerichteten und ausgeübten Gewerbebetrieb eine umfassende Interessenabwägung stattfinden, um die Rechtswidrigkeit eines Verhaltens feststellen zu können. Gleichwohl ist es sinnvoll, ein Recht am eingerichteten und ausgeübten Gewerbebetrieb anzuerkennen, um einen ersten Anhaltspunkt für die sorgfältige Prüfung zu haben, ob das Verhalten, das dieses Recht verletzt, rechtswidrig ist. Mit dieser beschränkten Indizwirkung erfüllt es im deutschen Recht eine sinnvolle Funktion. Es ist besser, eine schwache Indizwirkung zu haben als gar keine.

Andere Rechtssysteme gewähren in Boykottfällen, in Streikfällen, in Fällen des Warentest und anderen vergleichbaren Fällen ebenfalls Schadensersatz. Sie beurteilen die Rechtswidrigkeit aber, ohne ein Recht am eingerichteten und ausgeübten Gewerbebetrieb zugrunde zu legen, wie etwa das französische Recht bei Anwendung der Generalklausel des faute ${ }^{13}$. Im englischen Recht ist unsicher, inwieweit unter den Voraussetzungen von negligence der entgangene Gewinn ersetzt werden kann ${ }^{14}$. In anderen Fällen, die den Economic Torts zuzuordnen sind, ist für den Schadensersatzanspruch vorsätzliches und eine Art sittenwidrigen Verhaltens erforderlich ${ }^{15}$. Geht man davon aus, so fällt die Antwort schwer, ob das englische oder französische Recht bei Eingriffen Dritter in Systeme von Netz- oder Kettenverträgen Schadensersatz zubilligen würden.

11 Karl Larenz und Claus-Wilhelm Canaris (1994) Lehrbuch des Schuldrechts, 13. Aufl., München: Beck, § 81 III.

12 Von einer Schutzlücke geht auch BGHZ 45, 296, 307 aus.

13 Christian von Bar (1996) Gemeineuropäisches Deliktsrecht, München: Beck, Band I, Rn. $38 \mathrm{f}$.

14 Siehe von Bar (Fn. 13) Rn. 285 f.

15 Von Bar (Fn. 13) Rn. 260. 
Die deutsche Konzeption des Rechts am eingerichteten und ausgeübten Gewerbebetrieb gibt Anlass, näher nach dem geschützten Rechtsgut zu fragen. Je präziser dies festgestellt werden kann, desto einfacher ist es zu beurteilen, ob ein Eingriff in geschäftliche Aktivitäten rechtswidrig ist oder nicht. Es vereinfacht auch die Beurteilung, ob Netz- oder Kettenvertragssysteme ein über die bloßen Vertragsbeziehungen hinausgehendes Schutzgut haben. Wegen der Vorteile, die das Recht am eingerichteten und ausgeübten Gewerbebetrieb bietet, ist es nicht verwunderlich, dass der Große Zivilsenat des $\mathrm{BGH}$ erst kürzlich entschieden hat, dass er die fahrlässige falsche Schutzrechtsverwarnung weiterhin als eine Verletzung des Rechts am eingerichteten und ausgeübten Gewerbebetrieb ansehen will ${ }^{16}$.

In diesem Zusammenhang kann auch erwähnt werden, dass in der italienischen Rechtsordnung das Recht auf freie unternehmerische Initiative, das in etwa dem deutschen Recht am eingerichteten und ausgeübten Gewerbebetrieb entspricht, benutzt wird, um Schutz zu gewähren in Fällen, in denen mehrere Unternehmen bei ihrer Zusammenarbeit einem Boykott ausgesetzt sind ${ }^{17}$. Unter diesem Blickwinkel erscheint es nützlich zu fragen, ob Netzverträge oder gar Kettenverträge eine gemeinsame Verbindung zwischen ihren Mitgliedern aufweisen, die als Schutzgut gegen Eingriffe außen stehender Dritter angesehen werden könnte.

\section{Schutz von Netz- und Kettenverträgen gegenüber Dritten}

\section{Herkömmlicher Schadensersatz bei Eingriffen Dritter}

a) Betrachtet man zunächst das Franchisesystem, so kann man sicher feststellen, dass im Warentestfall der Franchisegeber Schadensersatz verlangen kann, weil sein Recht am eingerichteten und ausgeübten Gewerbebetrieb verletzt wurde. Nach allgemeiner Meinung umfasst der Schaden des Franchisegebers aber nur den entgangenen Gewinn seines eigenen Unternehmens einschließlich geringerer Einnahmen aus dem Umsatzrückgang der Franchisenehmer. Jedoch kann der Franchisegeber nur seinen eigenen, nicht den entgangenen Gewinn der Franchisenehmer aus deren Umsatzrückgang ersetzt verlangen. Mittelbar könnte der Franchisegeber den Schaden der Franchisenehmer ersetzt verlangen, wenn den Franchisenehmern ihrerseits Schadensersatzansprüche gegen den Franchisegeber zustünden. Dem Franchisegeber würde dann ein Schaden durch die Schadensersatzleistungen an die Franchisenehmer entstehen, den er ersetzt verlangen könnte. Aber nach deutschem Recht bestehen solche Schadensersatzansprüche nur, wenn der Franchisegeber vorsätzlich oder fahrlässig seine Vertragspflichten gegenüber den Franchisenehmern verletzt hätte. Bei einem falschen Warentest liegen solche Pflichtverletzungen aber nicht ohne weiteres vor. Deshalb bestehen vielfach keine Schadensersatzansprüche der Franchisenehmer gegen den Franchisegeber.

16 BGH GSZ vom 15.7.2005 GSZ 1/04 = NJW 2005, 3141.

17 Von Bar (Fn. 13) 62, Rn. 49. 
b) Dieselben Grundsätze gelten bei einem Streik im Unternehmen des Franchisegebers oder bei einem Streik im Unternehmen des Herstellers oder eines Lieferanten. Ohne vertragliche Beziehungen zum Schädiger und ohne Verletzung eines absoluten Rechts stehen den Geschädigten nach herrschender Meinung grundsätzlich keine Schadensersatzansprüche zu. Das BAG hat allerdings im Falle einer rechtswidrigen Betriebsblockade auch einem anderen Unternehmen, das nicht bestreikt, aber dennoch von der Blockade betroffen wurde, Schadensersatz zuerkannt ${ }^{18}$.

c) Gleiche Fragen stellen sich, wenn ein Dritter zu Unrecht eine Schutzrechtsverwarnung ausspricht und dadurch z. B. einen Produktionsstopp verursacht oder wenn ein Dritter die Marke oder ein anderes gewerbliches Schutzrecht des Franchisegebers oder des Herstellers verletzt und dadurch im Franchisesystem oder in der Organisation zwischen Hersteller und Lieferanten einen Gewinnausfall herbeiführt. Kann dann nur der Franchisegeber oder der Hersteller Schadensersatz verlangen oder können auch die Franchisenehmer oder die Lieferanten solche Ansprüche für die ihnen entstandenen Schäden geltend machen?

d) Bei Verletzung eines gewerblichen Schutzrechts durch einen Dritten ist auch der Lizenznehmer im Falle einer ausschließlichen Lizenz in seinen Rechten verletzt und kann deshalb Schadensersatz verlangen, nicht jedoch im Falle einer einfachen Lizenz. Etwas anderes gilt bei Verletzung eines Markenrechts. Das deutsche Markengesetz enthält spezielle Vorschriften. Nach $\S 30$ Abs. 3 MarkenG kann der Lizenznehmer vom Dritten Schadensersatz verlangen, gleichgültig ob es sich um eine ausschließliche oder nicht ausschließliche Lizenz handelt ${ }^{19}$. Jedoch kann der Lizenznehmer eine Klage gegen den Schädiger nur mit Zustimmung des Markeninhabers erheben. Wenn der Markeninhaber bereits Klage erhoben hat, kann der Lizenznehmer dieser Klage beitreten und Ersatz seines Schadens verlangen ( $\$ 30$ Abs. 4 MarkenG). Deshalb sind in Franchisesystemen die Franchisenehmer, die eine Lizenz an der Marke haben, ebenso wie der Franchisegeber geschützt. Sie können vom Schädiger auch ihre eigenen Schäden ersetzt verlangen und, wenn der Franchisegeber nicht seine Zustimmung zur Klageerhebung gibt, von diesem Schadensersatz verlangen. Insofern bedürfen die Franchisenehmer keines weiteren Schutzes. Wenn aber die Mitglieder eines Netzwerksystems keine Lizenz haben wie etwa in der Beziehung zwischen Hersteller und Lieferanten oder wenn die Lizenz nicht gegen Eingriffe Dritter geschützt ist, dann können nach derzeit vorherrschender Meinung die Mitglieder eines Netzwerksystems keinen Schadensersatz verlangen.

\section{Geschütztes Rechtssubjekt in Netz- und Kettenvertragssystemen}

Es stellt sich deshalb die Frage, wie die Mitglieder eines Netz oder Kettenvertragssystem geschützt werden können. Haben Netz- oder Kettenverträge einen über die bloß vertraglichen Beziehungen hinaus existierenden Schutzgegenstand, der wie ein abso-

18 BAG NJW 1989, 61.

19 Reinhard Ingerl und Christian Rohnke (2003) Markengesetz, 2 Aufl., München: Beck, § 30 Rn. 8 und 73. 
lutes Recht geschützt werden kann und der auch dem einzelnen Mitglied des Netzoder Kettenvertragssystems zugeordnet werden kann?

Obwohl die Franchisenehmer je für sich ein Recht am eingerichteten und ausgeübten Gewerbebetrieb haben, ist fraglich, ob ihr Recht in den genannten Fällen nach Auffassung des BGH verletzt wäre. Vertragliche Lieferbeziehungen sind nicht durch das Recht am eingerichteten und ausgeübten Gewerbebetrieb geschützt, weil es an einem betriebsbezogenen Eingriff fehlt. So stellt eine Störung in der Stromversorgung z. B. keinen betriebsbezogenen Eingriff dar $^{20}$.

Generell geht der BGH davon aus, dass jedes Unternehmen dem Wettbewerb ausgesetzt ist und die Beeinträchtigungen daraus hinnehmen muss ${ }^{21}$. Diese Grundsätze würden wohl auch bei einem falschen Warentest für die Franchisenehmer zur Anwendung gebracht werden. Das Gericht würde wahrscheinlich argumentieren, dass die Franchisenehmer ihren Geschäftsbetrieb ausüben können, indem sie ihre Waren von anderen Lieferanten beziehen. Außerdem würde das Gericht entsprechend herkömmlicher Meinung sicher davon ausgehen, dass die Idee, das Know-how und das ganze System des Franchisegebers durch das Recht am eingerichteten und ausgeübten Gewerbebetrieb geschützt wird und dass ein solches Recht auf Seiten der Franchisenehmer nicht verletzt sei. Aus denselben Gründen könnte das Recht am eingerichteten und ausgeübten Gewerbebetrieb nur beim bestreikten Unternehmen geschützt werden, nicht aber in anderen Unternehmen wie den Unternehmen der Franchisenehmer oder der Lieferanten, die nur mittelbar vom Streik im Unternehmen des Franchisegebers bzw. des Herstellers betroffen sind. Auch eine zu Unrecht erfolgte Schutzrechtsverwarnung würde nur den Franchisegeber oder Hersteller verletzen, der das Recht für sich in Anspruch nimmt, nicht aber die Franchisenehmer oder Lieferanten.

Jedoch lässt diese Auffassung die Tatsache außer Betracht, dass nicht nur der Franchisegeber und der Hersteller, sondern auch die Franchisenehmer und Lieferanten Teil des Netzwerksystems sind mit seiner auf Dauer eingerichteten Organisation, seinen Ideen, Markenrechten, dem Know-how einschließlich des Good will und der Wertschätzung der Kunden. Wäre das Unternehmen des Franchisenehmers Bestandteil des Unternehmens des Franchisegebers bzw. des Herstellers würden die Gerichte sicher den Schutz des Rechts am eingerichteten und ausgeübten Gewerbebetrieb gewähren. Es kann aber keinen Unterschied machen, wenn Unternehmensteile des Franchisegebers oder des Herstellers an Franchisenehmer oder Lieferanten als selbständige Unternehmen ausgelagert werden. Wer den falschen Warentest veröffentlicht oder den illegalen Streik organisiert, sollte nicht von der Auslagerung profitieren. Das wirtschaftliche Unternehmen im Ganzen ist in gleicher Weise beeinträchtigt, gleichgültig ob das Geschäft eine in das Unternehmen integrierte Filiale oder Niederlassung des Franchisegebers ist oder ob es ausgegliedert ist in ein (halb-)autonomes Geschäft des Franchisenehmers.

Die Gerichte würden wohl auch dem gesamten System Schutz gewähren und Schadensersatz zusprechen, wenn die Beziehung zwischen dem Franchisegeber und den

20 BGHZ 29, 65.

21 BGH NJW 1980, 881, 882. 
Franchisenehmern als Personengesellschaft ausgestaltet wäre ${ }^{22}$. Die rechtliche Konstruktion des Franchisesystems kann aber wiederum keinen Unterschied machen, soweit es die Zubilligung von Schadensersatzansprüchen betrifft. Die betroffenen Interessen sind immer die gleichen. Der Unterschied besteht allein darin, dass die verletzten Interessen im ersten Fall Bestandteil des einheitlichen Unternehmens des Franchisegebers und im zweiten Fall Teil des Unternehmens der Personengesellschaft sind, während die gleichen Interessen im dritten Fall unter mehrere (halb-)autonome Franchisenehmer aufgespalten sind. Das Schutzgut bleibt in allen Fällen das gleiche ${ }^{23}$.

Die Frage, ob die sozialen Beziehungen in einem Netzwerk wie dem Franchisesystem oder in einem Just in Time Liefersystem zwischen dem Hersteller und seinen Lieferanten als eine kollektive Einheit angesehen werden können, ist nicht nur für die Haftung der Systemmitglieder gegenüber Dritten von Bedeutung ${ }^{24}$, sondern auch für den Schutz des Systems gegenüber Angriffen von außen. An Stelle der Umgehung gesetzlicher Pflichten durch das System, der in ähnlicher Weise wie im Konzern ${ }^{25}$ mit Hilfe eines Durchgriffs durch das Netzwerk (piercing the network's veil) ${ }^{26}$ oder durch eine gesamtschuldnerische Haftung in hybriden Systemen begegnet werden kann ${ }^{27}$, erfordert der Schutz solcher Systeme die Suche nach gemeinsamen das System übergreifenden Rechtsgütern und Interessen, die allen Systemmitgliedern zugesprochen werden können und die als gemeinsame Rechtsgüter und Interessen aller Systemmitglieder gegen Angriffe von außen schützenwürdig sind.

\section{Das Marktmodell und das Modell der festen Lieferbeziehungen}

Der Grund dafür, dass die deutschen Gerichte Lieferbeziehungen gegen Beeinträchtigungen Dritter nicht schützen, liegt sicher darin, dass marktmäßige Lieferbeziehungen anders als Lieferbeziehungen innerhalb eines Unternehmens oder einer Personen- oder Kapitalgesellschaft nicht beständig sind, sondern dem Wettbewerb ausgesetzt sind und sich deshalb im Lauf der Zeit ändern können. Man kann dies das Marktmodell der Lieferbeziehungen nennen. Im Gegensatz dazu sind Lieferbeziehungen innerhalb eines Unternehmens oder eines Konzerns sorgfältig organisiert, um eine verlässliche und beständige Belieferung zu gewährleisten. Man kann dabei von einem Modell der festen Lieferbeziehungen sprechen.

Während eine Störung in den normalen Lieferbeziehungen zwischen Marktteilnehmern im allgemeinen leicht durch Suche nach einem anderen Anbieter am Markt ausgeglichen und behoben werden kann, kann eine Beeinträchtigung der Lieferbeziehung im Modell mit festen Lieferbeziehungen innerhalb eines Unternehmens oder eines

22 Von einer GbR als Schutzsubjekt geht BGH NJW 1992, 41 aus.

23 Ein ähnliches Argument findet sich bei Gunther Teubner (2004) Netzwerk als Vertragsverbund, Baden-Baden: Nomos, 223 ff. im Zusammenhang mit der Haftung der Mitglieder des Netzwerks.

24 Dazu Teubner (Fn. 23) $216 \mathrm{ff}$.

25 Teubner (Fn. 23) 220.

26 Analog zum "piercing the corporate veil"; Teubner (Fn. 23) 224, benutzt den Ausdruck piercing of the contractual veil.

27 Teubner (Fn. 23) 218 ff. 
Konzerns erhebliche Probleme verursachen, weil sich Zulieferer von außerhalb des Systems nicht ohne weiteres den speziellen Erfordernissen und Bedürfnissen des Produktionsprozesses anpassen können. Darüber hinaus ist eine erhebliche organisatorische Arbeit geleistet worden, um verlässliche und beständige Lieferbeziehungen innerhalb des Unternehmens oder Konzerns aufzubauen. Dies könnte nach Auffassung der deutschen Gerichte einen Schutz solcher gut organisierter ständiger betrieblicher Vorkehrungen mit Hilfe des Rechts am eingerichteten und ausgeübten Gewerbebetrieb rechtfertigen ${ }^{28}$. Die organisatorischen Schöpfungen und Leistungen des Unternehmers, die für das Funktionieren des Unternehmens wesentlich sind, sollten als der eigentliche Schutzbereich des Rechts am eingerichteten und ausgeübten Gewerbebetrieb anerkannt werden vergleichbar dem Schutz anderer Ideen und Leistungen, die Gegenstand der gewerblichen Schutzrechte und des Urheberrechts sowie des ergänzenden Leistungsschutzes im UWG sind ${ }^{29}$. Es ist deshalb zu fragen, ob Netz- und Kettenverträge eher dem Marktmodell oder dem Modell der festen Lieferbeziehungen zugeordnet werden sollen. Dies kann nur durch Prüfung einzelner Netz- und Kettenverträge entschieden werden.

\section{Das Franchisesystem als Modell der festen Lieferbeziehungen}

Die typischen Franchiseverträge können dem Modell mit festen Lieferbeziehungen zugeordnet werden. Der Franchisenehmer wird im allgemeinen durch den Franchisevertrag verpflichtet, die vom Franchisegeber angebotenen Waren und Leistungen vom Franchisegeber oder einem von diesem bestimmten Lieferanten zu beziehen. Der Franchisenehmer kann deshalb seine Lieferanten nicht frei am Markt auswählen, sondern muss sich auf das vom Franchisegeber angebotene Liefersystem stützen. Hinzukommt, dass der Franchisegeber große Anstrengungen unternommen hat, um das Liefersystem innerhalb des Franchisesystems aufzubauen. Dieses Liefersystem kann deshalb mit dem Liefersystem innerhalb eines Unternehmens oder innerhalb gesellschaftsrechtlich verbundener Unternehmen verglichen werden. Es kann deshalb dem Modell mit festen Lieferbeziehungen zugeordnet werden.

Daneben gibt es zusätzliche Gründe, das Franchisesystem durch das Recht am eingerichteten und ausgeübten Gewerbebetrieb zu schützen. Die Franchisenehmer benutzen z. B. die vom Franchisenehmer eingerichtete Marke oder sie profitieren von dessen Know-how ebenso wie von seiner Werbung zugunsten des Franchisesystems. Berücksichtigt man dies, so kann das Franchisesystem ohne weiteres mit einer Unternehmenseinheit verglichen werden, soweit es die wesentlichen Elemente des Rechts am eingerichteten und ausgeübten Gewerbebetrieb betrifft.

28 Die geschäftlichen Vorkehrungen werden hervorgehoben von BGH NJW 1961, 968; 1969 , 1207. - Die funktionellen Verbindungen der Produktionsmittel und Gegenstände im Unternehmen werden erwähnt in BGH NJW 1983, 812, 813.

29 Siehe $\S 4$ Nr. 9 UWG. 


\section{Das Just in Time Liefersystem als Modell der festen Lieferbeziehungen}

Dieselben Fragen stellen sich in den Beziehungen zwischen dem Hersteller und seinen Lieferanten im Just in Time Liefersystem. Bestehen hier ebenfalls so starke Verbindungen zwischen dem Hersteller und seinen Lieferanten, dass ihr System mit einem Unternehmen vor der Auslagerung von Betriebsteilen verglichen werden kann? Macht es z. B. einen Unterschied, wenn eine Produktionsstörung infolge eines Streiks vor der Auslagerung einzelner Teile des Produktionsprozesses oder erst danach stattfindet?

Bei Anwendung der Figur des Marktmodells und des Modells der festen Lieferbeziehungen ist es wiederum bedeutsam, ob die Vertragsbeziehungen zwischen dem Hersteller und seinen Lieferanten eine feste Verbindung mit ausschließlicher oder zumindest vorrangiger Lieferpflicht einerseits und eine ausschließliche oder zumindest überwiegende Bezugspflicht zugunsten eines bestimmten Lieferanten andererseits vorsehen. Besteht keine solch enge Beziehung zwischen Hersteller und Lieferanten, sondern kann jeder von ihnen sich selbst am Markt eindecken, so kann die Lieferbeziehung nicht mit den Lieferbeziehungen innerhalb eines Unternehmens verglichen werden und sollte nicht geschützt werden. Wenn aber eine feste Lieferbeziehung vergleichbar den Liefer- und Produktionsverhältnissen innerhalb eines Unternehmens besteht, dann sollte die Verbindung zwischen Hersteller und Zulieferer als eine Einheit betrachtet werden, soweit es den Schutz des Produktionsprozesses gegen einen illegalen Streik betrifft.

Konsequenz dieser Auffassung ist, dass z. B. ein Streik im Unternehmen des Herstellers auch den Zulieferer beeinträchtigen und zwingen kann, seine Produktion zu beschränken oder gar einzustellen so, als ob seine Produktion in das Unternehmen des Herstellers integriert wäre. Andererseits kann ein Streik im Unternehmen des Zulieferers den Hersteller ebenso treffen als ob der Streik in seinem eigenen Unternehmen stattfinden würde. Unter diesen Umständen sollte die feste Lieferbeziehung zwischen dem Hersteller und seinen Zulieferern so geschützt werden, als ob es eine einzige Unternehmenseinheit ohne Berücksichtigung der Auslagerung wäre.

Um den erforderlichen Schutz zu gewähren, sollte die Beziehung zwischen Hersteller und Zulieferer bei Just in Time Lieferungen wie eine eingerichtete und ausgeübte Geschäftseinheit behandelt werden, obwohl es eine Verbindung mehrerer Unternehmen und kein Einzelunternehmen ist. Mit der Anerkennung der Verbindung mehrerer Unternehmen als Inhaber des Rechts am eingerichteten und ausgeübten Gewerbebetrieb sollte auch der damit verbundene Schutz nach $\S 823 \mathrm{Ab}$. 1 BGB gewährt werden.

\section{Kettenverträge als Marktmodell}

Als Beispiel für einen Kettenvertrag kann eine Banküberweisung betrachtet werden, wenn zwei, drei oder mehr Banken hintereinander geschaltet sind, um die Überweisung auszuführen. Wenn eine Bank bei Durchführung der Überweisung ausfällt, sind die anderen Banken in der Kette nicht wirklich beeinträchtigt. Sie können ihren ganzen Geschäftsbetrieb und alle anderen Überweisungen ausführen. Ein Schaden entsteht nur beim Empfänger, der nicht über das Geld verfügen kann, oder es ist der Absender geschädigt, weil er Verzugszinsen zahlen muss. Dieselben Überlegungen 
gelten für Transportketten. Man kann deshalb sagen, dass bei den genannten Kettenverträgen und wahrscheinlich auch bei den meisten anderen Kettenverträgen kein gemeinschaftliches Schutzgut der Kettenmitglieder vorhanden ist, sondern alle nur individuell ihren Schaden geltend machen können.

\section{Schutz des Modells mit festen Lieferbeziehungen}

Bisher konnten zwei Fälle festgestellt werden, der Franchisevertrag und der Just in Time Liefervertrag, in denen ein Schutzgut entsprechend dem Recht am eingerichteten und ausgeübten Gewerbebetrieb vorhanden sein kann. Die Frage ist, was genau das Schutzgut in diesen Fällen ist, welches die einzelnen Bestandteile dieses Schutzguts sind und wem das damit begründete Recht am eingerichteten und ausgeübten Gewerbebetrieb zugeordnet werden kann.

\section{Die Organisation des Netzvertragssystems als geschütztes Rechtsgut}

Die wesentlichen Elemente eines Franchisesystems, die das Recht am eingerichteten und ausgeübten Gewerbebetrieb begründen, stimmen mit den wesentlichen Charakteristika von Franchisesystemen überein, wie die Marke und andere gewerbliche Schutzrechte des Franchisegebers, die alle Franchisenehmer nutzen dürfen, das Know-how, das der Franchisegeber den Franchisenehmern zur Verfügung stellt, der gemeinsame Name, unter dem alle Franchisenehmer am Markt auftreten dürfen, der Good-will, der sich auf diesen Namen stützt, die Werbemaßnahmen des Franchisegebers und schließlich die gesamte vom Franchisegeber aufgebaute Organisation. Obwohl diese Bestandteile des Franchisesystems in erster Linie dem Franchisegeber zuzuordnen sind, erleiden auch die Franchisenehmer Schäden, wenn diese Schutzgüter durch Dritte verletzt werden. Deshalb sollte das ganze System als ein allumfassendes Rechtsgut unter Einschluss der Franchisenehmer geschützt werden.

Ähnliche Überlegungen können bei der Beziehung zwischen Hersteller und Zulieferern angestellt werden. Wenn die Lieferbeziehungen hier so eng organisatorisch verbunden sind, wie in einem Einzelunternehmen, so sollte diese organisatorische Einheit auch durch das Recht am eingerichteten und ausgeübten Gewerbebetrieb geschützt werden, als ob es ein einheitliches Unternehmen wäre, auch wenn es sich um verschiedene Unternehmen und keine gesellschaftsrechtliche Verbindung handelt. Gleiches sollte beim organisatorischen Zusammenwirken von General- und Subunternehmer sowie beim Reisevertrag nach § 651a BGB gelten. Wer aber kann als Inhaber dieses Rechts angesehen werden?

\section{Bruchteilsgemeinschaft zwischen Mitgliedern des Netzvertragssystems}

Das Franchisesystem, das aus dem Franchisegeber und vielen Franchisenehmern besteht, kann hinsichtlich der Zuordnung von Rechten nicht wie ein Einzelkaufmann oder eine juristische Person behandelt werden. Das Franchisesystem kann auch nicht wie eine Personengesellschaft geschützt werden, weil nicht wie bei der Gesellschaft vertragliche Beziehungen zwischen den Franchisenehmern untereinander bestehen. Es 
gibt auch kein Gesamthandsvermögen, was für die Personengesellschaften charakteristisch ist. Das deutsche Recht bietet aber die Bruchteilsgemeinschaft als Rechtsinstitut, das für die Rechtszuordnung im Franchisesystem benutzt werden kann. Der Franchisegeber und jeder Franchisenehmer ist danach zu einem Bruchteil an dem Recht am eingerichteten und ausgeübten Gewerbebetrieb beteiligt, welches das Franchisesystem als organisatorische Einheit aller Beteiligten schützt. Das Schutzgut der organisatorischen Einheit zwischen dem Hersteller und seinen Zulieferern im Just in Time System kann den Systemmitgliedern ebenfalls in Bruchteilsgemeinschaft zugeordnet werden. Die Franchisenehmer und Zulieferer als Systemmitglieder ermächtigen dabei mit ihrem Systembeitritt den Franchisegeber und Hersteller als Systemführer, weitere Beteiligte in die Bruchteilsgemeinschaft hinsichtlich des Rechts am eingerichteten und ausgeübten Gewerbebetrieb aufzunehmen. Vergleichbare Überlegungen können für Beziehung zwischen General- und Subunternehmer sowie beim Reise vertrag gemäß $\S$ 651a BGB zur Anwendung kommen.

\section{Schadensersatz für Beeinträchtigungen des Netzvertragssystems}

Aufgrund der Bruchteilsgemeinschaft hinsichtlich des Rechts am eingerichteten und ausgeübten Gewerbebetrieb kann das einzelne Systemmitglied Schadensersatz verlangen, wenn das System z. B. durch einen falschen Warentest, durch eine falsche Schutzrechtsverwarnung, durch einen illegalen Streik, durch Schmähkritik oder durch einen Boykottaufruf rechtswidrig beeinträchtigt wird. Derselbe Schutz sollte dem Franchisenehmer und dem Zulieferer in der Hersteller-Zulieferer Beziehung gewährt werden, wenn die Marke oder andere gewerbliche Schutzrechte des Franchisegebers oder Herstellers verletzt werden. Auch wenn die Franchisenehmer oder Zulieferer keine Lizenz an der Marke des Franchisegebers oder Herstellers haben, sind sie doch beeinträchtigt, weil durch eine Beeinträchtigung der Marke oder durch einen Umsatzrückgang beim Hersteller auch ihr Umsatz und Gewinn zurückgehen kann. Der Dritte, der die Marke des Franchisegebers oder Herstellers verletzt, hat für die Folgen dieser Verletzung einzustehen und kann nicht davon profitieren, dass der Franchisenehmer und der Zulieferer ausgelagert sind und kein integraler Bestandteil der Firma des Franchisegebers oder des Herstellers sind. An Stelle eines integrierten Einzelunternehmens tritt die beständig eingerichtete Organisation des Franchisesystems oder des Hersteller-Zulieferer Netzvertragssystems. Diese Überlegungen können wiederum entsprechend auf die Beziehung zwischen General- und Subunternehmer und den Reisevertrag gemäß $§ 651$ a BGB übertragen werden.

Jedes Mitglied des betreffenden Systems kann aber nur seinen eigenen Schaden ersetzt verlangen und nicht den Schaden des gesamten Systems. Die Franchisenehmer können deshalb nur Ersatz des ihrer Einzelfirma entstandenen Schadens verlangen und der Franchisegeber kann nur für die von ihm erlittenen Schäden Ersatz verlangen, z. B. für die Kosten, um das Franchisesystem wieder aufzubauen, oder für den seinem Unternehmen entgangenen Gewinn. In gleicher Weise kann der Hersteller und die Zulieferer nur Ersatz des ihnen jeweils selbst entstandenen Schadens verlangen.

Es könnte eingewandt werden, dass der Schädiger übermäßig belastet werden könnte, wenn man jedes Systemmitglied zu einem Bruchteil am Recht am eingerichte- 
ten und ausgeübten Gewerbebetrieb beteiligt und ihm einen entsprechenden Schadensersatzanspruch zuerkennt, weil dann der Schädiger mehreren oder gar allen Systemmitgliedern Schadensersatz leisten müsste. Aber dem ist zu entgegnen, dass der Schaden derselbe oder zumindest vergleichbar bleibt gegenüber der Beziehung zwischen sich beliefernden Unternehmensteilen in ein Einheitsunternehmen, wenn die Systemmitglieder in das Einheitsunternehmen eingegliedert und nicht ausgelagert wären. Darüber hinaus muss der Schädiger auf die Güter und Rechte anderer Rücksicht nehmen und kann nicht verlangen, dass er aufgrund seines rechtswidrigen Verhaltens nur dem einen zufällig ausgegliederten Unternehmen verantwortlich sein soll.

\section{Schlussbemerkungen}

Die vorstehend aufgezeigte Konzeption ist sicherlich progressiv und wird in der vorherrschenden Doktrin nicht vertreten. Aber neue Erscheinungen verlangen neue Lösungen. Deshalb sollte die Erweiterung und zugleich Wiederbelebung des Rechts am eingerichteten und ausgeübten Gewerbebetrieb als eine realistische Lösung in Betracht gezogen werden. Netzvertragsysteme und möglicherweise auch - wenngleich in geringerem Maße - Kettenvertragssysteme sollten gegen Störungen Dritter von außen durch das Recht am eingerichteten und ausgeübten Gewerbebetrieb geschützt werden, wenn sie als System mit festen Lieferbeziehungen organisiert sind, so dass ihre innere Struktur so aufgebaut ist, dass Systemmitglieder durch vertragliche Beziehungen fest und ständig miteinander verbunden und deshalb voneinander abhängig sind. 